\title{
Comparison of Survival Between Primary Debulking Surgery Versus Neoadjuvant Chemotherapy for Ovarian Cancers in a Personalized Treatment Cohort
}

Zheng Feng ${ }^{1,2 t}$, Hao Wen ${ }^{1,2 t}$, Ruimin $\mathrm{Li}^{2,3}$, Shuai $\mathrm{Liu}^{2,4,5,6}$, Yi Fu ${ }^{2,3}$, Xiaojun Chen ${ }^{1,2}$, Rui $\mathrm{Bi}^{2,7}$, Xingzhu $\mathrm{Ju}^{1,2^{*}}$ and Xiaohua $\mathrm{Wu}^{1,2^{*}}$

\section{OPEN ACCESS}

Edited by:

Giuseppe Vizzielli,

Catholic University of the Sacred

Heart, Italy

Reviewed by:

Martina Arcieri,

University of Messina, Italy

Raffaella Ergasti,

A. Gemelli University Hospital

Foundation (IRCCS), Italy

${ }^{*}$ Correspondence: Xingzhu Ju

xingzi_ju@163.com

Xiaohua Wu

docwuxh@hotmail.com;

wu.xh@fudan.edu.cn

${ }^{t}$ These authors have contributed equally to this work

Specialty section: This article was submitted to Women's Cancer,

a section of the journal

Frontiers in Oncology

Received: 22 November 2020 Accepted: 21 December 2020 Published: 10 February 2021

Citation:

Feng Z, Wen H, Li R, Liu S, Fu Y, Chen X, Bi R, JU X and Wu X (2021) Comparison of Survival Between Primary Debulking Surgery Versus

Neoadjuvant Chemotherapy for Ovarian Cancers in a Personalized Treatment Cohort.

Front. Oncol. 10:632195. doi: 10.3389/fonc.2020.632195

\begin{abstract}
${ }^{1}$ Department of Gynecological Oncology, Fudan University Shanghai Cancer Center, Shanghai, China, ${ }^{2}$ Department of Oncology, Shanghai Medical College, Fudan University, Shanghai, China, ${ }^{3}$ Department of Radiology, Fudan University Shanghai Cancer Center, Shanghai, China, ${ }^{4}$ Department of Nuclear Medicine, Fudan University Shanghai Cancer Center, Shanghai, China, ${ }^{5}$ Center for Biomedical Imaging, Fudan University, Shanghai, China, ${ }^{6}$ Shanghai Engineering Research Center of Molecular Imaging Probes, Fudan University, Shanghai, China, ${ }^{7}$ Department of Pathology, Fudan University Shanghai Cancer Center, Shanghai, China
\end{abstract}

Objective: To compare survival between primary debulking surgery (PDS) and neoadjuvant chemotherapy (NACT) for the treatment of ovarian cancer patients per our selective protocol.

Methods: Between Sep $1^{\text {st }}, 2015$, and Aug $31^{\text {st }}, 2017,161$ patients were enrolled in our prospective cohort. All of the patients received preoperative clinic-radiological assessments, according to the Suidan criteria for $\mathrm{RO}$ resection. Patients with a score of $0-2$ received PDS. Patients with a score of $\geq 3$ were counseled on the choices of PDS, NACT, or an optional staging laparoscopy, according to the Fagotti criteria. Clinic-pathological data were prospectively collected until May $1^{\text {st }}, 2020$, and the impacts of different treatment strategies on progression-free survival (PFS) and overall survival (OS) were analyzed.

Results: 110 patients underwent PDS, and 51 patients received NACT with consequent interval debulking surgery. The R0 resection rate was $57.8 \%$. All but one of the patients received platinum-based chemotherapy, and 105 (65.2\%) patients were platinumsensitive. Based on the univariate analysis, the PDS group exhibited prolonged PFS compared with the NACT group ( $P=0.029)$. The subgroup analysis showed that patients receiving NACT with residual disease $(R D)$ exhibited the worst PFS $(P=0.001)$. Based on the multivariate analysis, NACT with RD was still an independent impaired factor for PFS $(P=0.04)$. However, NACT did not affect OS in the univariate or multivariate analyses.

Conclusion: In our prospective cohort, NACT ovarian patients exhibited inferior PFS and noninferior OS compared with PDS patients. Given our selective protocol, NACT cannot be arbitrarily denied while appropriate PDS is still a priority.

Keywords: ovarian cancer, primary debulking surgery, neoadjuvant chemotherapy, residual disease, progressionfree survival, overall survival 


\section{INTRODUCTION}

Ovarian cancer (OC) continues to be the most lethal disease among females worldwide (1), and the five-year overall survival rate is lower than 50\% (2). Primary debulking surgery (PDS) and individual platinum-based adjuvant chemotherapy are the standard treatments for ovarian cancer patients. However, neoadjuvant chemotherapy (NACT) has been developed as an alternative for PDS.

Two randomized trials (EORTC 55971 and CHORUS) have shown noninferior prognoses with a lower risk for postoperative adverse events in NACT groups compared with that in PDS groups of advanced OC patients (3-5). However, these noninferior results of the NACT groups (compared to PDS groups) was not confirmed in the JCOG0602 trial (6). Although results demonstrating that NACT can equate to PDS are conflicting, it is well acknowledged that patients with complete resection at PDS have the best prognoses. Thus, the use of NACT has been proposed for OC patients with disseminated, unresectable disease. In addition, appropriate selection criteria are urgently required for treatment strategies for OC patients.

Since 2015, the specialized ovarian cancer unit at our institution has implemented a personalized surgical approach for the treatment of OC patients (7). We adopted two-tier predictive models for $\mathrm{R} 0$ resection; specifically, a preoperative clinic-radiological assessment for all OC patients and a laparoscopic assessment, when necessary. In this paper, the authors report on the updated data from the study, which compared the survival between PDS and NACT groups of enrolled OC patients per our selective protocol.

\section{METHODS}

\section{Data Collection}

This study was conducted according to the Declaration of Helsinki and was approved by the Committee at Fudan University Shanghai Cancer Center. All of the individual participants consented to the use of their medical records for research purposes.

Between Sep $1^{\text {st }}, 2015$, and Aug $31^{\text {st }}, 2017,161$ OC patients were enrolled in our prospective personalized treatment cohort. All of the patients had preoperative clinic-radiological assessments, according to the Suidan criteria for R0 resection (8). Patients with a score of 0-2 were determined to have PDS. Patients with a score of $\geq 3$ were counseled on the choices of PDS, NACT, or further laparoscopic assessments, according to the Fagotti criteria (9).

The pathological diagnoses were reviewed (according to the WHO criteria) by two experienced gynecological pathologists. Additionally, the patients were staged according to the 2014 FIGO criteria. R0 was defined as the absence of macroscopic residual disease $(\mathrm{RD})$ after surgery. According to the response to the platinum-based chemotherapy, patients were classified as being either platinum-sensitive or platinum-resistant $(10,11)$. Progression-free survival (PFS) was defined as the interval ranging from the date of the primary surgery or the first cycle of neoadjuvant chemotherapy to the date of disease progression or recurrence. Overall survival (OS) was defined as the interval ranging from the date of the primary surgery or the first cycle of neoadjuvant chemotherapy to the date of death or the last followup (May $1^{\text {st }}, 2020$ ).

\section{Statistical Analyses}

SPSS statistical software (version 26.0, SPSS, IBM Inc., New York, USA) was used for the statistical analyses. Descriptive statistics were used for the demographic data, and the data were summarized as medians with ranges or frequencies with percentages. The PFS and OS were analyzed with the KaplanMeier method and with log-rank tests in the univariate analyses. For the multivariate analyses, a Cox regression analysis was used to evaluate the effects of the prognostic factors, which were expressed as hazard ratios (HRs). $\mathrm{P}<0.05$ was considered to be statistically significant, and all of the reported $\mathrm{P}$ values were 2 -sided.

\section{RESULTS}

\section{Patient Characteristics}

The patient characteristics are summarized in Table 1. The median (range) age was 57 (27-77)-years-old. Among the 161 OC patients who were enrolled, 110 patients underwent PDS, and 51 received NACT, with corresponding interval debulking surgery (IDS). The majority $(158 / 161,98.1 \%)$ of the patients were of an advanced FIGO stage, including 128 patients with stage III disease and 30 patients with stage IV disease. The R0 resection rate was $57.8 \%$ in the whole cohort. Detailed residual disease data were shown previously. That is, $62(56.4 \%)$ patients in the PDS group had no residual disease, while 31 (28.2\%) and $17(15.5 \%)$ patients received $\mathrm{R} 1$ and $\mathrm{R} 2$ resections, respectively. In the NACT group, $60.8 \%$ (31/51) of patients had R0 resection, while the R1 and R2 rates were 29.4\% (15/51) and 9.8\% (5/51), respectively. All but one of the patients received platinum-based chemotherapy, and 105 (65.2\%) patients were platinum-

TABLE 1 | Patient characteristics $(n=161)$

\begin{tabular}{llcc}
\hline Age & Median (range) & \multicolumn{2}{c}{$\mathbf{5 7}$ (27-77) } \\
\hline FIGO Stage & Early & 3 & $1.9 \%$ \\
& Advanced & 158 & $98.1 \%$ \\
& Stage III & 128 & $79.5 \%$ \\
Family history & Stage IV & 30 & $18.6 \%$ \\
\multirow{2}{*}{ Treatment strategy } & Yes & 54 & $33.5 \%$ \\
& No & 107 & $66.5 \%$ \\
Residual disease & PDS & 110 & $68.3 \%$ \\
Platinum sensitivity & NACT+IDS & 51 & $31.7 \%$ \\
& RO & 93 & $57.80 \%$ \\
& RD & 68 & $42.20 \%$ \\
Status & Yes & 105 & $65.2 \%$ \\
& No & 48 & $29.8 \%$ \\
& NA & 8 & $5.0 \%$ \\
& Alive & 108 & $67.10 \%$ \\
& Dead & 50 & $31.10 \%$ \\
& Censored & 3 & $1.90 \%$
\end{tabular}


sensitive. Besides, 91 patients in our cohort had germline $B R C A$ mutation tests. Among them, 31 patients harbored deleterious $B R C A$ mutations.

\section{Prognostic Impacts of the Different Treatment Strategies}

The median follow-up time was 38 (1-53) months. The median (95\% confidence interval, CI) PFS was 18 (14.6-21.3) months. Six patients in the PDS group died within half a year. Among them, four patients received $\mathrm{R} 0$ resections, and died of severe perioperative complications. While another two patients had bulky residual disease, and died of disease progression. Furthermore, the histological types of two patients indicated clear cell cancer. Twenty-two (13.7\%) women experienced disease progression during adjuvant chemotherapy, and eightyseven $(54.0 \%)$ patients exhibited documented recurrence. The recurrence patterns between the two groups are shown in Figure 1. Eleven patients had secondary cytoreductive surgery with R0 resection. Eight platinum-sensitive recurrent patients had PARP inhibitor maintenance, including six with niraparib and two with olaparib. The median OS was not able to be estimated. One hundred and eight (67.1\%) patients were still alive at the time of the last follow-up, and fifty (31.1\%) deaths were documented.

The known negative effects of platinum resistance on PFS $(\mathrm{P}<0.001)$ and $\mathrm{OS}(\mathrm{P}<0.001)$ were confirmed. Although patients with clinic-radiological scores of $0-2$ had prolonged PFS and OS, compared to patients with a score of $\geq 3$, the differences were not significant $(\mathrm{P}=0.147$ and $\mathrm{P}=0.441$, respectively).

Figure 2 shows the PFS according to the treatment arm and debulking results. Based on the univariate analysis, it was demonstrated that the PDS group had prolonged PFS compared with the NACT group, with median (95\% CI) PFS
A

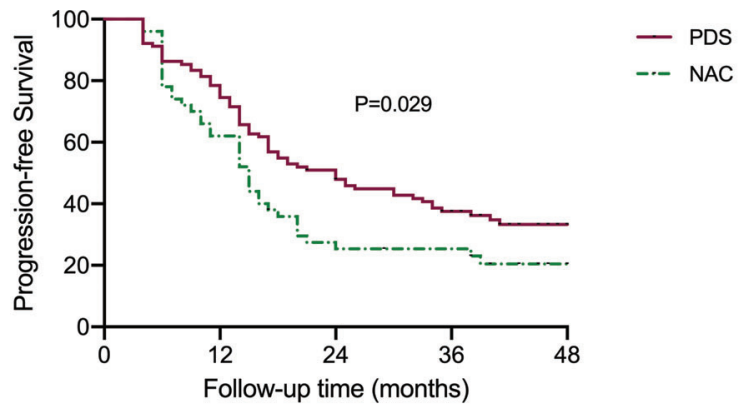

B

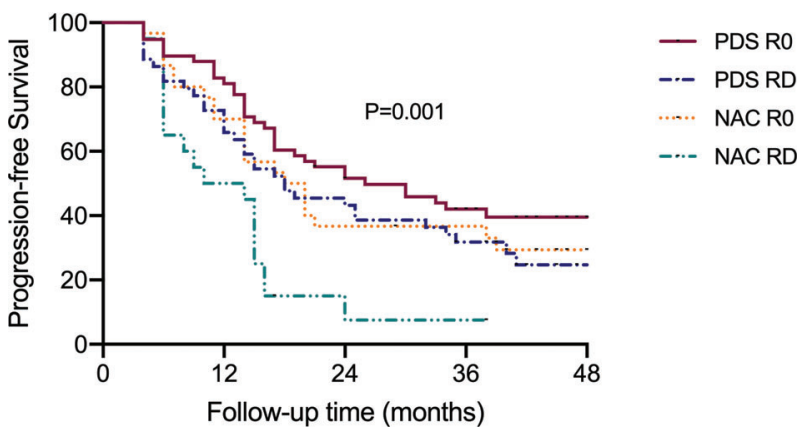

FIGURE 2 | Progression-free survival of patients according to the subgroups. (A) Stratified by different treatment strategies. (B) Stratified by treatment strategies and residual disease.
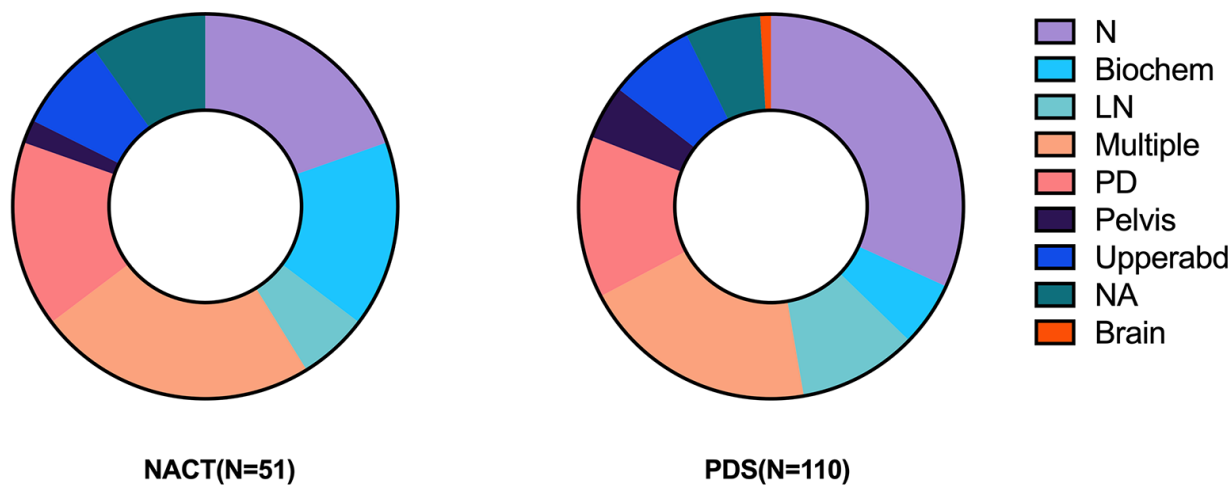

\begin{tabular}{|c|c|c|c|c|c|c|c|c|}
\hline Groups & N & Biochemical & Lymphnode & Multiple & PD & Pelvis & Upperabdominal & NA \\
\hline PDS & 35 & 6 & 11 & 22 & 15 & 5 & 8 & 7 \\
\hline NACT & 10 & 8 & 3 & 12 & 8 & 1 & 1 & 4 \\
\hline
\end{tabular}

FIGURE 1 | Recurrence patterns of disease between the NACT and PDS groups. 
values of 24.0 (16.7-31.3) months and 15.0 (11.9-18.1) months, respectively ( $\mathrm{P}=0.029$, Figure $\mathbf{2 A}$ ). The median (95\% CI) PFS values for patients with R0 resection at PDS, residual disease at PDS, R0 resection at IDS and residual disease at IDS were 26.0 (12.1-39.9) months, 18.0 (8.7-27.3) months, 18.0 (11.6-24.4) months, and $10.0(0-20.96)$ months, respectively. The subgroup analysis showed that patients with residual disease who received NACT had the worst PFS ( $\mathrm{P}=0.001$, Figure 2B). Based on the multivariate analysis, it was demonstrated that NACT with residual disease was still an independent impaired factor for PFS $[\mathrm{HR}=2.011$ (1.031-3.923), $\mathrm{P}=0.04$, Table 2].

Figure 3 shows the OS according to the treatment arm and the residual disease status. Based on the univariate analyses, it was demonstrated that neither the different treatment strategy nor the residual disease status affected $\mathrm{OS}(\mathrm{P}=0.433$ and $\mathrm{P}=0.330$, respectively). Additionally, there was no difference among the groups that were stratified by residual disease and treatment strategy subclassification (Table 3).

\section{DISCUSSION}

In our prospective cohort, we validated two-tier predictive models for R0 resection to determine if OC patients were to receive PDS or NACT. After a greater than three-year follow-up period, we demonstrated that NACT ovarian patients had inferior PFS, when compared with PDS patients. However, there was no difference in the OS between the two groups.

As we have previously reported, our two-tier predictive algorithm is convenient for OC treatment decision-making (7). The R0 rate of debulking surgery has been improved to $57.8 \%$ compared with the rate of $31 \%$ based on our historical data (10). Additionally, these results are comparable to those from other studies, which have reported R0 rates ranging from $41 \%$ to $67.8 \%$ (11-13). Given our prospective feature, the data from our personalized cohort were objective and reliable.

The choice of NACT as an effective alternative for PDS of OC treatment has been debated for years. Two randomized trials (EORTC 55971 and CHORUS) have reported that patients in the NACT group achieved higher R0 rates and lower perioperative complication rates, when compared to those patients in the PDS group $(3,5)$. Furthermore, there were no survival differences between the two groups. However, the R0 rates in the PDS

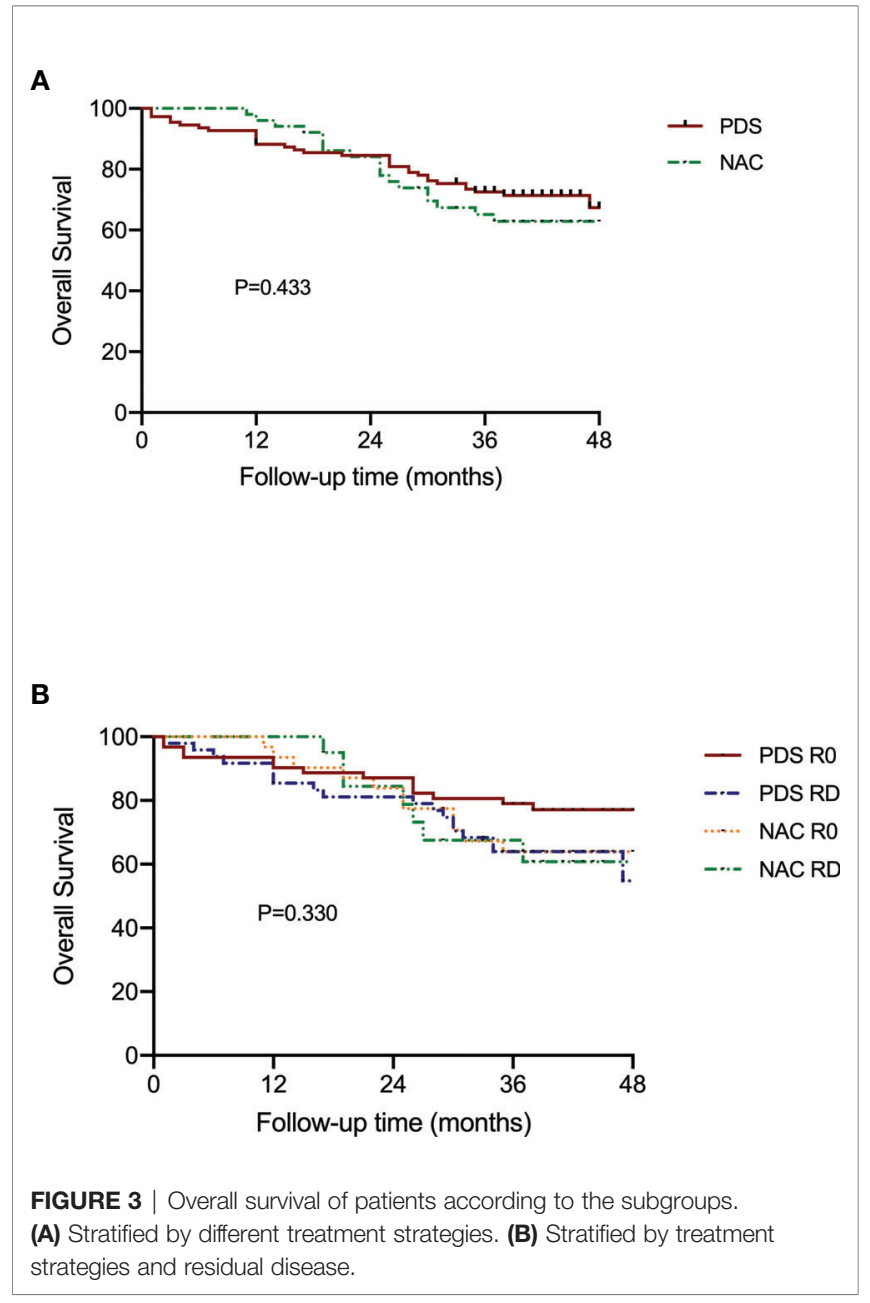

groups were below $20 \%$, which were much lower compared with the rates in other studies $(14,15)$, and it is not clear whether maximal efforts were performed during PDS. Thus, several scholars have challenged the concept of substituting NACT for PDS. Onda et al. (6) have reported of another noninferior phase III randomized trial to compare PDS and NACT in OC treatment. In this study, a survival noninferiority effect of NACT was not confirmed (compared with PDS), with median OS values of 44.3 and 49.0 months, respectively.

TABLE 2 | Cox regression analysis of PFS.

\begin{tabular}{|c|c|c|c|c|c|c|}
\hline \multirow[t]{2}{*}{ Characteristics } & & \multicolumn{5}{|c|}{ os } \\
\hline & & HR & & $95 \% \mathrm{Cl}$ & & $P$ value \\
\hline Age (continuous variable) & & 0.970 & 0.950 & - & 0.990 & 0.004 \\
\hline \multirow[t]{2}{*}{ Platinum sensitivity } & No & & & Referent & & \\
\hline & Yes & 0.014 & 0.006 & - & 0.031 & $<0.001$ \\
\hline \multirow[t]{4}{*}{ Patterns of residual disease } & PDS with $\mathrm{RO}$ resection & & & Referent & & \\
\hline & PDS with RD & 1.448 & 0.882 & & 2.377 & 0.143 \\
\hline & NACT with R0 resection & 1.209 & 0.666 & - & 2.194 & 0.532 \\
\hline & NACT with RD & 2.011 & 1.031 & - & 3.923 & 0.040 \\
\hline \multirow[t]{2}{*}{ Clinic-radiological score } & $0-2$ & & & Referent & & \\
\hline & $\geq 3$ & 0.575 & 0.346 & - & 0.955 & 0.033 \\
\hline
\end{tabular}


TABLE 3 | Cox regression analysis of OS.

\begin{tabular}{|c|c|c|c|c|c|c|}
\hline \multirow[t]{2}{*}{ Characteristics } & & \multicolumn{5}{|c|}{ os } \\
\hline & & HR & & $95 \% \mathrm{Cl}$ & & $P$ value \\
\hline Age (continuous variable) & & 0.970 & 0.940 & - & 1.000 & 0.051 \\
\hline \multirow[t]{2}{*}{ Platinum sensitivity } & No & & & Referent & & \\
\hline & Yes & 0.049 & 0.022 & - & 0.108 & $<0.001$ \\
\hline \multirow[t]{4}{*}{ Patterns of residual disease } & PDS with R0 resection & & & Referent & & \\
\hline & PDS with RD & 1.769 & 0.787 & & 3.977 & 0.167 \\
\hline & NACT with R0 resection & 2.054 & 0.806 & - & 5.231 & 0.131 \\
\hline & NACT with RD & 0.663 & 0.237 & - & 1.858 & 0.435 \\
\hline \multirow[t]{2}{*}{ Clinic-radiological score } & $0-2$ & & & Referent & & \\
\hline & $\geq 3$ & 0.710 & 0.323 & - & 1.562 & 0.395 \\
\hline
\end{tabular}

In our cohort, the patients in the NACT groups exhibited impaired PFS, when compared to those patients in the PDS group, whereas there were no survival differences from the OS analysis. In contrast from the previously mentioned randomized trials, our prospective cohort demonstrated a validated two-tier algorithm for treatment strategy determination. As a result, the patients with a higher tumor burden tended to be allocated to the NACT group. Thus, we used the clinic-radiological score as an indicator for tumor burden in the multivariate analyses. It was demonstrated that NACT with residual disease was still an independent impaired factor. In regard to the OS analysis, although nearly five years had passed since the first patient's enrollment, $67.1 \%$ patients were still currently alive, and the median OS was not able to be estimated. Thus, further follow-ups are needed.

Furthermore, it should be noted that six patients in the PDS group died within half a year. Among them, four patients had R0 resections with extreme debulking surgery and perioperative complications. Similar to the results of the SCORPION trial, over half of the OC patients with a high tumor burden who received PDS experienced perioperative moderate or severe morbidities $(16,17)$. Patients with high tumor load had worst prognosis, while there was no prognostic difference between PDS and NACT groups for this subgroup $(18,19)$. Narasimhulu et al. (20). demonstrated the use of the Mayo triage algorithm to identify OC patients who were at the highest risk of morbidity and mortality after debulking surgery. They included indicators such as albumin, age, and presumed surgical complexity to determine the uses of either upfront surgery or chemotherapy. Thus, the decision on whether to use PDS or NACT for OC patients should consider not only the resectability of the tumor burden but also the tolerability of the patients. NACT could be a candidate for OC patients who cannot afford extensive surgical procedures to achieve a complete cytoreduction.

In addition, 11 patients received secondary cytoreductive surgeries with R0 resection. The recent data of Desktop III have shown as surgery resection can improve the prognosis. Marchetti $\mathrm{C}$ et al. (21) also reported the benefit of secondary cytoreduction even in $B R C A$ mutated patients with olaparib maintenance. Besides, eight platinum-sensitive recurrent patients had PARP inhibitor maintenance. And these above might also influence the overall survival analysis.

In conclusion, NACT ovarian patients exhibited inferior PFS compared with PDS patients in our prospective cohort. In addition, the OS data require further follow-ups. Given the nature of our selective protocol, NACT cannot be arbitrarily denied while appropriate PDS is still a priority.

\section{DATA AVAILABILITY STATEMENT}

The raw data supporting the conclusions of this article will be made available by the authors, without undue reservation.

\section{ETHICS STATEMENT}

The studies involving human participants were reviewed and approved by the Committee at Fudan University Shanghai Cancer Center. The patients/participants provided their written informed consent to participate in this study.

\section{AUTHOR CONTRIBUTIONS}

ZF and HW participated in the study design, carried out the data collection, performed the statistical analysis, and drafted the manuscript. RL, SL, and YF carried out the data collection and participated in the radiological review. $\mathrm{RB}$ participated in the pathologic review of all slides. XC carried out the data collection. $\mathrm{XJ}$ and XW conceived the study and participated in its design and coordination. All authors read and approved the final manuscript. All authors contributed to the article and approved the submitted version.

\section{FUNDING}

This work was financially supported through a grant from the leading project of the Science and Technology Commission of Shanghai Municipality (No. 19411960200) for XW.

\section{ACKNOWLEDGMENTS}

The authors would like to thank all of the doctors, nurses, patients, and their family members for their support of the present study. 


\section{REFERENCES}

1. Torre LA, Bray F, Siegel RL, Ferlay J, Lortet-Tieulent J, Jemal A. Global cancer statistics, 2012. CA Cancer J Clin (2015) 65(2):87-108. doi: 10.3322/caac.21262

2. Berek JS, Kehoe ST, Kumar L, Friedlander M. Cancer of the ovary, fallopian tube, and peritoneum. Int J Gynaecol Obstet (2018) 143 Suppl 2:59-78. doi: 10.1002/ijgo. 12614

3. Kehoe S, Hook J, Nankivell M, Jayson GC, Kitchener H, Lopes T, et al. Primary chemotherapy versus primary surgery for newly diagnosed advanced ovarian cancer (CHORUS): an open-label, randomised, controlled, noninferiority trial. Lancet (2015) 386(9990):249-57. doi: 10.1016/S0140-6736 (14)62223-6

4. Vergote I, Amant F, Kristensen G, Ehlen T, Reed NS, Casado A. Primary surgery or neoadjuvant chemotherapy followed by interval debulking surgery in advanced ovarian cancer. Eur J Cancer (2011) 47 Suppl 3:S88-92. doi: 10.1016/S0959-8049(11)70152-6

5. Vergote I, Trope CG, Amant F, Kristensen GB, Ehlen T, Johnson N, et al. Neoadjuvant chemotherapy or primary surgery in stage IIIC or IV ovarian cancer. N Engl J Med (2010) 363(10):943-53. doi: 10.1056/NEJMoa0908806

6. Onda T, Satoh T, Ogawa G, Saito T, Kasamatsu T, Nakanishi T, et al. Comparison of survival between primary debulking surgery and neoadjuvant chemotherapy for stage III/IV ovarian, tubal and peritoneal cancers in phase III randomised trial. Eur J Cancer (2020) 130:114-25. doi: 10.1016/j.ejca.2020.02.020

7. Feng Z, Wen $\mathrm{H}$, Jiang Z, Liu S, Ju X, Chen $\mathrm{X}$, et al. A triage strategy in advanced ovarian cancer management based on multiple predictive models for R0 resection: a prospective cohort study. J Gynecol Oncol (2018) 29(5):e65. doi: 10.3802/jgo.2018.29.e65

8. Suidan RS, Ramirez PT, Sarasohn DM, Teitcher JB, Iyer RB, Zhou Q, et al. A multicenter assessment of the ability of preoperative computed tomography scan and CA-125 to predict gross residual disease at primary debulking for advanced epithelial ovarian cancer. Gynecol Oncol (2017) 145(1):27-31. doi: 10.1016/j.ygyno.2017.02.020

9. Fagotti A, Ferrandina G, Fanfani F, Ercoli A, Lorusso D, Rossi M, et al. A laparoscopy-based score to predict surgical outcome in patients with advanced ovarian carcinoma: a pilot study. Ann Surg Oncol (2006) 13 (8):1156-61. doi: 10.1245/ASO.2006.08.021

10. Feng Z, Wen H, Bi R, Ju X, Chen X, Yang W, et al. A clinically applicable molecular classification for high-grade serous ovarian cancer based on hormone receptor expression. Sci Rep (2016) 6:25408. doi: 10.1038/srep25408

11. Rutten MJ, van Meurs HS, van de Vrie R, Gaarenstroom KN, Naaktgeboren CA, van Gorp T, et al. Laparoscopy to Predict the Result of Primary Cytoreductive Surgery in Patients With Advanced Ovarian Cancer: A Randomized Controlled Trial. J Clin Oncol (2017) 35(6):613-21. doi: 10.1200/JCO.2016.69.2962

12. Aletti GD, Garbi A, Messori P, Achilarre MT, Zanagnolo V, Rizzo S, et al. Multidisciplinary approach in the management of advanced ovarian cancer patients: A personalized approach. Results from a specialized ovarian cancer unit. Gynecol Oncol (2017) 144(3):468-73. doi: 10.1016/j.ygyno.2017.01.017

13. Fagotti A, Vizzielli G, Fanfani F, Costantini B, Ferrandina G, Gallotta V, et al. Introduction of staging laparoscopy in the management of advanced epithelial ovarian, tubal and peritoneal cancer: impact on prognosis in a single institution experience. Gynecol Oncol (2013) 131(2):341-6. doi: 10.1016/j.ygyno.2013.08.005

14. Chi DS, Eisenhauer EL, Lang J, Huh J, Haddad L, Abu-Rustum NR, et al. What is the optimal goal of primary cytoreductive surgery for bulky stage IIIC epithelial ovarian carcinoma (EOC)? Gynecol Oncol (2006) 103(2):559-64. doi: 10.1016/j.ygyno.2006.03.051

15. Fagotti A, Vizzielli G, Fanfani F, Scambia G. Comparison of peritoneal carcinomatosis scoring methods in predicting resectability and prognosis in advanced ovarian cancer. Am J Obstet Gynecol (2010) 203(3):e10-1; author reply e1. doi: 10.1016/j.ajog.2010.04.037

16. Fagotti A, Ferrandina G, Vizzielli G, Fanfani F, Gallotta V, Chiantera V, et al. Phase III randomised clinical trial comparing primary surgery versus neoadjuvant chemotherapy in advanced epithelial ovarian cancer with high tumour load (SCORPION trial): Final analysis of peri-operative outcome. Eur J Cancer (2016) 59:22-33. doi: 10.1016/j.ejca.2016.01.017

17. Vizzielli G, Costantini B, Tortorella L, Pitruzzella I, Gallotta V, Fanfani F, et al. A laparoscopic risk-adjusted model to predict major complications after primary debulking surgery in ovarian cancer: A single-institution assessment. Gynecol Oncol (2016) 142(1):19-24. doi: 10.1016/j.ygyno.2016.04.020

18. Fagotti A, Ferrandina MG, Vizzielli G, Pasciuto T, Fanfani F, Gallotta V, et al. Randomized trial of primary debulking surgery versus neoadjuvant chemotherapy for advanced epithelial ovarian cancer (SCORPIONNCT01461850). Int J Gynecol Cancer (2020) 30(11):1657-64. doi: 10.1136/ ijgc-2020-001640

19. Vizzielli G, Costantini B, Tortorella L, Petrillo M, Fanfani F, Chiantera V, et al. Influence of intraperitoneal dissemination assessed by laparoscopy on prognosis of advanced ovarian cancer: an exploratory analysis of a singleinstitution experience. Ann Surg Oncol (2014) 21(12):3970-7. doi: 10.1245/ s10434-014-3783-6

20. Narasimhulu DM, Kumar A, Weaver AL, Langstraat CL, Cliby WA. Less guessing, more evidence in identifying patients least fit for cytoreductive surgery in advanced ovarian cancer: A triage algorithm to individualize surgical management. Gynecol Oncol (2020) 157(3):572-7. doi: 10.1016/ j.ygyno.2020.03.024

21. Marchetti C, Rosati A, Scaletta G, Pietragalla A, Arcieri M, Ergasti R, et al. Secondary cytoreductive surgery in platinum-sensitive recurrent ovarian cancer before olaparib maintenance: Still getting any benefit? A case-control study. Gynecol Oncol (2019) 155(3):400-5. doi: 10.1016/j.ygyno.2019.09.020

Conflict of Interest: The authors declare that the research was conducted in the absence of any commercial or financial relationships that could be construed as a potential conflict of interest.

Copyright (C) 2021 Feng, Wen, Li, Liu, Fu, Chen, Bi, Ju and Wu. This is an open-access article distributed under the terms of the Creative Commons Attribution License (CC BY). The use, distribution or reproduction in other forums is permitted, provided the original author(s) and the copyright owner(s) are credited and that the original publication in this journal is cited, in accordance with accepted academic practice. No use, distribution or reproduction is permitted which does not comply with these terms. 